\title{
Aporte de las revistas científicas colombianas de teología y filosofía en la difusión de investigaciones sobre corrupción, paz y reconciliación a partir de un estudio bibliométrico*
}

\section{Flor Alba Acuña}

Magíster en Teología de la Universidad Pontifica Bolivariana, especialista en Gestión de la Investigación de la Universidad Manuela Beltrán, teóloga de la Fundación Universitaria Seminario Bíblico de Colombia (FUSBC), bacterióloga y laboratorista clínica de la Universidad Industrial de Santander. Profesora del área de Bibliae investigación de la FUSBC,

Medellín-Colombia.

facuna@unisbc.edu.co

orcid.org/0000-0003-1301-3380

\section{Magle Virginia Sánchez Castellanos Doctora en Ciencias de la Educación de la Universidad Dr. Rafael Belloso Chacin, magíster en Ciencias Gerenciales de la Universidad Gran Mariscal de Ayacucho e ingeniera de Computación de la Universidad Rafael Urdaneta. Profesora en el área de Informática Educativa y Metodología de la Investigación en la Universidad Cooperativa de Colombia. Bogotá-Colombia. \\ magle.sanchez@campusucc.edu.co orcid.org/0000-0003-0650-355X}

\begin{abstract}
Resumen
Resumen

El objetivo del presente artículo fue identificar el aporte de las revistas científicas colombianas de teología y filosofía en la difusión de investigaciones relacionadas con los temas de corrupción, paz y reconciliación, por medio de un estudio bibliométrico centrado en indicadores de impacto con el propósito de generar reflexiones. El método se centró en un estudio cuantitativo no experimental, siguiendo el método de revisión sistemática. En Colombia, existen nueve revistas científicas en el área de teología y filosofía indexadas en Publindex; el 11,1 \% está en categoría A2, el 22,2 \% en B y el $66,7 \%$ en C. En cuanto a la posición en SJR, el 22,2 \% está en los cuartiles medios (Q2 y Q3), el 33,3\% en Q4 y el 44,4 \% sin cuartil. Respecto a la difusión relacionada con los temas de corrupción, paz y reconciliación, el aporte es muy bajo: 3,4 \% y 2,7 \% respectivamente. La baja visibilidad de estas publicaciones plantea la necesidad de evaluar el cumplimiento de gestión editorial y calidad de contenido, que afectan la divulgación de los resultados de investigación, pero también se debe tener en cuenta las características y dinámicas propias de las publicaciones en humanidades.
\end{abstract}

Palabras clave: corrupción, paz y reconciliación, factores de impacto, análisis de contenido, teología, filosofía.

Cómo citar este artículo: Acuña, F., \& Sánchez, M. (2019). Aporte de las revistas científicas colombianas de teología y filosofía en la difusión de investigaciones sobre corrupción, paz y reconciliación a partir de un estudio bibliométrico. Revista Interamericana de Bibliotecología, 42(2), 127-139. doi: 10.17533/udea.rib.v42n2a02

Recibido: 2018-05-28 / Aceptado: 2019-03-20

\footnotetext{
* El presente texto es un avance del proyecto "Estudio comparativo de los criterios de calidad y condiciones editoriales de la revista académico científico de Colombia en relación con las revistas mejor categorizadas a nivel nacional e internacional" del Grupo de Investigación Informática Educativa de la Universidad Manuela Beltrán, Av. Circunvalar N.o 60-00 Bogotá- Colombia.
} 


\section{Contribution of the Colombian Scientific Journals of Theology and Philosophy in the Dissemination of Research on Corruption, Peace and Reconciliation Based on a Bibliometric Study}

\begin{abstract}
The objective of this article is to identify the contribution of scientific journals of Theology and Philosophy to the dissemination of research related to corruption, peace and reconciliation issues, through a bibliometric study focused on quality criteria with the purpose of generate reflections. The method focusing on a non-experimental quantitative study, following the method of systematic review. In Colombia, there are 11 scientific journals in the area of Theology and Philosophy indexed in IBNPublindex, $11.1 \%$ are in category A2, $22.2 \%$ in B, and $66.7 \%$ in C. Regarding the position in SJR, $22.2 \%$ are in the middle quartiles (Q2 and Q3), 33.3\% in Q4 and $44.4 \%$ without quartile. With respect to corruption, peace and reconciliation, the contribution is very low, $3.4 \%$ and $2.7 \%$ respectively. The low visibility of these publications. Information. Information. The publications. The answers. Humanities.
\end{abstract}

Keywords: Corruption, peace and reconciliation, quality, content analysis, Theology, Philosophy.

\section{Introducción}

Las revistas científicas y especializadas son uno de los principales medios de difusión de la producción intelectual, científica y académica de investigadores e instituciones; producen un impacto académico, social y económico capaz de generar innovación y progreso de la ciencia y la sociedad; ellas visibilizan "la calidad de los avances en las diferentes disciplinas en las que se investiga" (Ramírez, Martínez \& Castellanos, 2012, p. 46). Para lograr lo anterior, estas revistas deben cumplir parámetros de calidad de contenido, visibilidad e impacto.
Al analizar la visibilidad de las revistas iberoamericanas, Penkova (2011) afirma que estas se caracterizan por la "poca difusión y visibilidad..., deficientes esquemas de gestión de la información académica y científica (...), necesidad de evaluación pertinente de la producción científica regional" (p. 99). En cuanto a prácticas y políticas editoriales de revisión por pares, el estudio realizado por Tavares (2011) mostró que las revistas científicas internacionales del área de humanidades y ciencias sociales ofrecen poca información detallada sobre las mismas, con promedios bajos, siendo la media del 5,6 \% y 9,7\% respectivamente.

Debido a este panorama y teniendo en cuenta el momento actual que está viviendo Colombia ante la lucha contra la corrupción (como antivalor) y la búsqueda de mecanismos para construir paz y reconciliación (como valores esenciales) luego de la firma del Acuerdo de Paz entre el Estado y las Fuerzas Armadas Revolucionarias de Colombia (FARC), la presente investigación pretendió identificar el aporte de las revistas científicas colombianas de teología y filosofía en la difusión de investigaciones relacionadas con los temas de corrupción, paz y reconciliación, por medio de un estudio bibliométrico centrado en indicadores de impacto con el propósito de generar reflexiones.

El artículo está organizado en cuatro secciones. En la primera sección se desarrollan los referentes teóricos; en la segunda se describe la metodología del estudio, y en la tercera se presentan los resultados. Por último, se señalan la discusión de los hallazgos y las conclusiones.

\section{Referentes teóricos}

Colombia, desde hace varias décadas, atraviesa por un conflicto armado con diferentes aristas y motivos, pero luego de la firma del Acuerdo de Paz se hace meritorio el estudio y análisis de dos temas relevantes: la corrupción, como antivalor, y la paz y reconciliación, como valores de gran significado para el país. Dichos estudios pueden ser llevados a cabo desde posturas teológicas, de esta manera, se prestaría atención a la actividad humana "a partir de la realidad concreta para transformarla bajo el impulso de la palabra, que se hace acción posibilitadora del Reino de Dios en la historia" (López, 2015 , p. 2), brindando y perfilando un sentido o revelación desde doctrinas sagradas, relaciones espirituales 
y divinas, interés intelectual, responsabilidad social, ética y tensiones dialécticas, lo que conlleva temas filosóficos. En este último particular, para Cortés (2016):

La finalidad de los estudios de paz no es la neutralidad descriptiva de los fenómenos sociales, sino la gestión no violenta de los conflictos sociales y el diseño de modelos de convivencia basados en el respeto, la tolerancia y el diálogo. (p. 81)

De esta manera, estudios bajo estas áreas pueden brindar valiosas contribuciones que permitan entender la dinámica social de Colombia, para mejorar el manejo de los conflictos sociales, permitiendo resaltar valores y dejando a un lado los antivalores.

En este mismo orden de ideas, la corrupción, como antivalor, es un fenómeno que afecta a las sociedades de todo el mundo y obstaculiza el progreso de las naciones. "No solo acarrea serias consecuencias éticas y morales, sino que genera graves distorsiones económicas, sociales y políticas, afectando la gobernabilidad de los países y poniendo en riesgo la viabilidad democrática de los estados" (Sánchez, 2015, p. 370).

El informe presentado en 2017 por la organización no gubernamental Transparencia Internacional ${ }^{1}$ muestra que la gran mayoría de los países hispanoparlantes y Brasil, en América Latina, se encuentran entre los más corruptos del mundo, debido a que poseen un Índice de Percepción de Corrupción (IPC) por debajo del valor medio, el cual es de 43/100. Los países que tienen un mejor índice son Uruguay y Chile, con 70 y 67 puntos respectivamente, mientras que Nicaragua y Venezuela poseen los peores índices, con 26 y 18 puntos (Transparencia Internacional, 2017). Estos resultados muestran que aunque la mayoría de los países de América Latina están luchando contra la corrupción, siguen teniendo altos niveles y continúan siendo asolados por el soborno y la impunidad.

1 Organización creada en 1993, la cual promueve medidas contra los crímenes corporativos y la corrupción política en el ámbito internacional. A través de estudios comparativos mide el índice de percepción de corrupción en el sector público de 180 países y territorios del mundo clasificándolos según su prevalencia de corrupción en una escala de 0 a 100, en la que 0 es altamente corrupto y 100 muy limpio.
Según este informe, Colombia ha mantenido su IPC en 37 puntos en los últimos cuatro años (2017-2014), mismo puntaje que Brasil, Panamá y Perú ((Transparencia Internacional, 2017). A pesar de los esfuerzos del Gobierno nacional y del sector privado por controlar la corrupción, la percepción de esta problemática no ha cambiado. En este mismo sentido, en Colombia predominan, entre otros, el cohecho, el peculado, la malversación de recursos, el soborno, la apropiación de bienes públicos y privados, el enriquecimiento ilícito y la celebración indebida de contratos. Gómez (2018) afirma que prácticas como el clientelismo y el favorecimiento judicial potencian las redes sistémicas y las prácticas de corrupción. En un comunicado, Transparencia por Colombia (2017) propone tres importantes reformas para el país: "transformar el sistema político y el ejercicio del poder; recuperar la legitimidad de la justicia y lograr sanciones efectivas; y romper con el clientelismo en el empleo público y la contratación" (s. p.).

Por otro lado, Colombia lleva más de cincuenta años en confrontación armada, siendo los principales actores de tal conflicto grupos al margen de la ley y el Estado. El Centro Nacional de Memoria Histórica (CNMH, 2013) afirma que este conflicto es uno de los más sangrientos en América Latina y que, entre los factores determinantes y recurrentes del origen y la perduración del mismo, se reconocen los problemas vinculados a la tierra, la precariedad y debilidad de la democracia y la impunidad. La magnitud de esta confrontación armada es tal que ha generado no solo víctimas mortales, sino también daños a la población con graves consecuencias como desaparecidos, víctimas de violencia sexual, reclutamiento de niños, niñas y adolescentes, y desplazamiento forzado, convirtiendo a Colombia en el primer país a nivel mundial con la mayor cantidad de desplazados internos y el segundo con el mayor número de víctimas de minas antipersona, después de Afganistán.

Toda esta situación atenta contra la integridad física, la libertad y los bienes de los ciudadanos y los lleva a la desolación, la desesperanza, la deshumanización y la perdida de dignidad, por lo que el llamado a la paz y reconciliación es urgente e imperativo.

A partir del 2012, se ha buscado la paz y reconciliación a través de las negociaciones con las FARC, de igual forma, se iniciaron intentos por restablecer los derechos de las víctimas a través de políticas de reparación inte- 
gral. Luego de firmar un acuerdo de paz entre el Estado y las FARC, uno de los mayores retos para construir la paz en Colombia es buscar mecanismos para la reconciliación. Para Barreto (2017), la reconciliación es un proceso complejo y multidimensional que se da tanto a nivel social (Estado y sociedad) como a nivel comunitario e individual. Este proceso implica coexistencia pacífica, confesión, perdón, reconocimiento del valor y la dignidad del otro, reintegración y reconfiguración de relaciones sociales.

Debido a las consecuencias que han generado tanto la corrupción como el conflicto armado en Colombia, hay una necesidad relevante de buscar la paz y la reconciliación, pero esta búsqueda puede tener diferentes protagonistas, formas y perspectivas, entre estas, las llevadas a cabo desde el seno de las instituciones de educación superior (IES). En las IES, destaca la función sustantiva de la investigación, cuya acción se divulga y queda en manifiesto, entre otros, a través de producciones bibliográficas de tipo artículos plasmados en revistas científicas, las cuales permiten la visibilización de las investigaciones en la sociedad científica.

En Colombia, el Departamento Administrativo de Ciencia, Tecnología e Innovación (Colciencias) creó el Sistema Nacional de Indexación y Homologación de Revistas Especializadas de CTeI (Publindex), y el Índice Bibliográfico Nacional (IBN), el cual analiza las revistas científicas para reconocerlas y clasificarlas en categorías (Al, A2, B y C), de acuerdo con exhaustivos criterios de gestión editorial, calidad de contenido y visibilidad, reconocidos a nivel internacional para publicaciones científicas (Colciencias, 2016).

Aunque en los últimos años se ha evidenciado un incremento de la producción y del número de revistas científicas nacionales, "solo el 13,8\% se encontraban incluidas en los índices bibliográficos citacionales (IBC) del SCI y Scopus" (Colciencias, 2016, p. 12), lo que refleja una escasa contribución a la visualización del conocimiento nacional en ámbitos internacionales.

Por otro lado, en un mundo que genera constantemente información científica interdisciplinaria, las bases de datos juegan un rol primordial en el incremento de la difusión y visibilidad de esta información, contenida en revistas y artículos publicados por estas bases. Buscando tener una mayor visibilidad, las revistas tienden a indexar- se en bases de datos internacionales, de las cuales, las más prestigiosas son Web of Science (WoS), de Thomson Reuters, y Scopus, de Elsevier. También se encuentra Google Scholar de Google Inc., la cual es reconocida por su poder de búsqueda. Estas bases de datos tienen sus propios requisitos y criterios de calidad, para la selección de revistas que desean indizarse en ellas.

En pro de la calidad, las revistas científicas deben cumplir con un conjunto de parámetros y criterios editoriales que tienen que ver con la calidad de contenido, la gestión y política editorial, la difusión, la estabilidad, la visibilidad e impacto, para asegurar el rigor científico de los artículos (Cardinali, 2010; Penkova, 2011). Entre estos parámetros destaca la visibilidad, que se evalúa "tanto por el alcance de la difusión de las revistas como por la recepción que la comunidad científica dispensa a los artículos publicados en ellas, a través de la citación" (Miguel, 2011, p. 188).

Actualmente, la visibilidad es medida a través de indicadores bibliométricos que son usados para evaluar la producción y citación de las investigaciones científicas, y se basan en "el número de citas que reciben las revistas científicas (en un período determinado y en función del número de artículos que se publican)" (Buela-Casal, 2003, p. 24). El indicador más reconocido e influyente es el Factor de impacto (FI), pero también ha sido uno de los más cuestionados en los últimos tiempos, en cuanto a su objetividad para la medición de la producción científica (Hicks, Wouters, Waltman, De Rijcke \& $\&$ Rafols, 2015; Buela-Casal, 2003). Sin embargo, siguiendo la figura de impacto se han creado otros indicadores, entre ellos, el Source-Normalized Impact per Paper (SNIP), el SCImago Journal Rank (SJR), el Eigenfactor, el Article Influence y, el más reciente, el índice h. En la siguiente tabla (Tabla l) se explican diferentes factores de impacto, tanto el más influyente como los que se utilizaron en este estudio.

El FI es un índice bibliométrico que permite evaluar y comparar las producciones científicas de un país, así, permite conocer el nivel de difusión entre la comunidad científica (Buela-Casal, 2003). Los factores de impacto mencionados (tanto el original como sus variaciones) permiten brindar un ponderado tanto a revistas como a autores, para futuramente determinar grados de difusión e impacto de lo publicado. De igual forma, este ponderado puede aplicarse a un país y a un tema en específico, para determinar o identificar su difusión. 
Tabla 1. Indicadores de impacto de las publicaciones científicas.

\begin{tabular}{|c|c|c|}
\hline Indicador & Cálculo & Características \\
\hline $\begin{array}{l}\text { Factor de Impacto (FI). } \\
\text { Eugene Garfield, } 1955\end{array}$ & $\begin{array}{l}\text { Se divide "el número de citaciones recibidas por los } \\
\text { artículos de esa revista en los dos años inmediatamente } \\
\text { anteriores, por el número total de publicaciones de } \\
\text { la revista durante ese mismo periodo de tiempo" } \\
\text { (Colciencias, 2016, p. 5). }\end{array}$ & $\begin{array}{l}\text { "Mide la influencia e impacto de una revista científica } \\
\text { en su categoría temática, y muestra las relaciones } \\
\text { existentes entre citación y revistas citadas, asignando } \\
\text { cuartiles a las revistas que integran cada una de las } \\
\text { categorías" (Colciencias, 2016, p. 24). Se utilizó por } \\
\text { primera vez para cuantificar las publicaciones en la } \\
\text { edición de } 1963 \text { del Science Citation Index (SCI). Es } \\
\text { calculado por Thomson Reuters a través del Journal } \\
\text { Citation Reports (JCR) de Web of Knowledge. }\end{array}$ \\
\hline SCImago Journal Rank (SJR) & $\begin{array}{l}\text { Calcula el peso de las citas según el indicador SJR de } \\
\text { la revista que cita, de esta manera tiene en cuenta las } \\
\text { citas recibidas por parte de las revistas más influyentes. } \\
\text { Usa el método de cálculo de PageRankTM. Excluye } \\
\text { autocitaciones superiores al } 33 \% \text {, y su ventana de citación } \\
\text { es de } 3 \text { años. (González, Guerrero \& Moya, 2010) }\end{array}$ & $\begin{array}{l}\text { Mide tanto el prestigio como el impacto de los artículos } \\
\text { de las revistas científicas. Al utilizar una ventana } \\
\text { de citación de tres años cubre el rango de citas de un } \\
\text { número significativo de revistas y refleja la dinámica de } \\
\text { la comunicación científica. Su fuente de información } \\
\text { son las revistas indexadas en la base de datos de Scopus } \\
\text { de Elsevier. Calculado por Scimago Lab. Plataforma } \\
\text { SCImago Journal \& Country Rank (http://www. } \\
\text { scimagojr.com/). }\end{array}$ \\
\hline Índice h. Jorge Hirsch, 2005 & $\begin{array}{l}\text { Se organizan en orden descendente los artículos del autor } \\
\text { según el número de citaciones recibidas, se numera esta } \\
\text { lista y "se desciende hasta identificar el último punto en } \\
\text { el que el número de orden coincide o es menor al número } \\
\text { de citaciones correspondiente" (Ramírez et al., 2012, p. } \\
\text { 108) }\end{array}$ & $\begin{array}{l}\text { Un científico tiene índice h si el h de sus trabajos (Np) } \\
\text { recibe al menos h citas cada uno, y los otros (Np - h) } \\
\text { trabajos tienen como máximo h citas cada uno (Hirsch, } \\
\text { 2005, p. 16569). En las plataformas SJR y Google Scholar } \\
\text { se presentan el índice h de cada revista. El índice H } \\
\text { "es la forma más objetiva de evaluar el impacto de la } \\
\text { producción científica de un investigador" (Díaz, 2014,p. } \\
\text { l14) porque mide el impacto global de sus publicaciones } \\
\text { indexadas en Scopus o Web of Knowledge. El índice } \\
\text { h-5 "restringe el cálculo de las citaciones recibidas } \\
\text { por una publicación científica en los últimos } 5 \text { años, } \\
\text { independientemente de su fecha de publicación" } \\
\text { (Colciencias, 2016, p. 6). }\end{array}$ \\
\hline
\end{tabular}

Fuente: adaptado de Ramírez et al., 2012.

\section{Método}

El presente estudio se desarrolló bajo un enfoque cuantitativo y un diseño no experimental, para su realización se siguió el método de revisión sistemática de Kitchenham (2004). La población y muestra seleccionadas estuvo centrada solo en publicaciones científicas nacionales que se encuentran en revistas colombianas de teología y filosofía. El estudio estuvo conformado por las siguientes fases:

\subsection{Fase 1. Planificación}

\subsubsection{Selección de revistas.}

Con el fin de conocer el número de publicaciones que existen en el área de teología y filosofía en Colombia, se hizo un rastreo en las páginas web de las instituciones universitarias que cuentan con facultades de teología y humanidades. Posteriormente, para la selección definitiva de las revistas científicas nacionales de teología y filosofía se constató su existencia o indización en la base de datos IBN-Publindex.

\subsubsection{Criterios de inclusión de revistas.}

Teniendo en cuenta el objetivo de la investigación, se definió como criterio de inclusión ser una revista científica en el área de teología o filosofía reconocida y categorizada por Publindex, en la Convocatoria 768 de 2016 en sus dos etapas.

\subsection{Fase 2. Revisión}

\subsubsection{Extracción de datos.}

Inicialmente, de las revistas seleccionadas, se extrajeron los siguientes datos: nombre de la revista, año 
de fundación, periodicidad, números publicados, descripción de la publicación, institución editora y categoría en Publindex. Posteriormente, se examinó la visibilidad de estas revistas en bases de datos y sus factores de impacto.

Para identificar la actividad científica relacionada con los temas de corrupción, paz y reconciliación, se realizó un análisis de contenido y así se pudo determinar el número de artículos escritos, por cada año de edición, sobre estas dos temáticas. En el análisis, se revisaron los resúmenes de cada uno de los artículos publicados, con una ventana de observación de tres años (2015-2017) y se consideraron palabras clave en cada una de las temáticas propuestas. En la temática de corrupción las palabras fueron corrupción, ética de principios, crisis, soborno e integridad, y para el tema de paz y reconciliación, las palabras objeto de estudio fueron reconciliación, paz disminución del conflicto, violencia, diálogo, justicia y equidad.

\subsubsection{Síntesis de datos.}

Los datos identificados se sintetizaron en tablas para su posterior análisis.

\subsection{Fase 3. Reporte y análisis}

\subsubsection{Reporte.}

Una vez terminada la extracción y síntesis de datos se procedió a realizar el reporte y análisis crítico basados en los criterios de impacto establecidos por IBN-Publindex, con el fin de establecer el aporte en la difusión de las investigaciones relacionadas con corrupción, paz y reconciliación en las revistas académicas en Colombia en el campo de teología y filosofía.

\section{Resultados}

El estudio se inició con una revisión en los portales institucionales de las instituciones universitarias que cuentan con facultades de teología y humanidades y se logró identificar 13 revistas nacionales que publican resultados de investigación en el área de teología, de las cuales cinco estaban categorizadas en el IBN-Publindex (antes de la segunda etapa del 2017), así: Theologica Xaveriana (A2), Frasciscanum (A2), Cuestiones Teológicas (B), Albertus Magnus (C) y Perseitas (C) y ocho no estaban indexadas. Al realizar la búsqueda en la base de datos IBN-Publindex, luego de la segunda etapa de 2017, se seleccionaron las revistas de teología y filosofía que cumplieron con los criterios de inclusión, estas se relacionan en la Tabla 2.

Dada la información anterior, en Colombia existen nueve revistas científicas en el área de teología y filosofía (clasificadas por IBN-Publindex), a través de las cuales se difunden los resultados de investigación tanto a nivel nacional como internacional.

La Tabla 2 permite observar que de las nueve revistas que cumplieron los criterios exigidos por el IBN-Publindex el 11,1 \% (1) es de teología y el 88,9 \% (8) de filosofía. En cuanto a la categorización, el 11,1 \% (1) está en categoría A2, el 22,2 \% (2) en categoría B y el 66,7 \% restante (6) en C. Además, cinco de ellas son editadas por instituciones universitarias privadas y cuatro por instituciones públicas. Todas las revistas (el $100 \%$ ), declaran su periodicidad: el 88,9\% (8) es semestral y el $11,1 \%$ (1) es cuatrimestral.

De igual forma, la antigüedad de las revistas varía entre ellas: el 22,2 \% (2 - Theologica Xaveriana e Ideas y Valores) con más de sesenta años, ambas fundadas en 1951 y poseen los más altos números de publicaciones, 184 y 165, respectivamente; el 11,1\% (1-Escritos) entre cuarenta y sesenta años, con 55 números publicados; el 33,3\% (3-Cuadernos de Filosofía, Estudios de Filosofía y Folios) entre veinte y treinta y nueve años y el restante 33,3\% (3-Discusiones Filosóficas, Eidos y Co-herencia) con menos de veinte años, con un promedio de 28 números publicados cada uno.

En cuanto al tipo de publicación que hacen, todas afirman que publican artículos inéditos resultado de investigación en el área de las humanidades, específicamente en los campos de teología (Theologica Xaveriana) y filosofía, pero algunas, como las revistas Co-herencia, Folios y Escritos, incluyen trabajos en otros campos como literatura, lingüística, idiomas, historia, geografía, estudios clásicos, teoría política y poesía. Otras mencionan que también incluyen artículos de reflexión y revisión, reseñas de libros, ensayos, traducciones y ponencias.

En la Tabla 3, se presentan la visibilidad e impacto de las nueve revistas de teología y filosofía clasificadas en el IBN-Publindex. 
Tabla 2. Revistas científicas de teología y filosofía en Colombia.

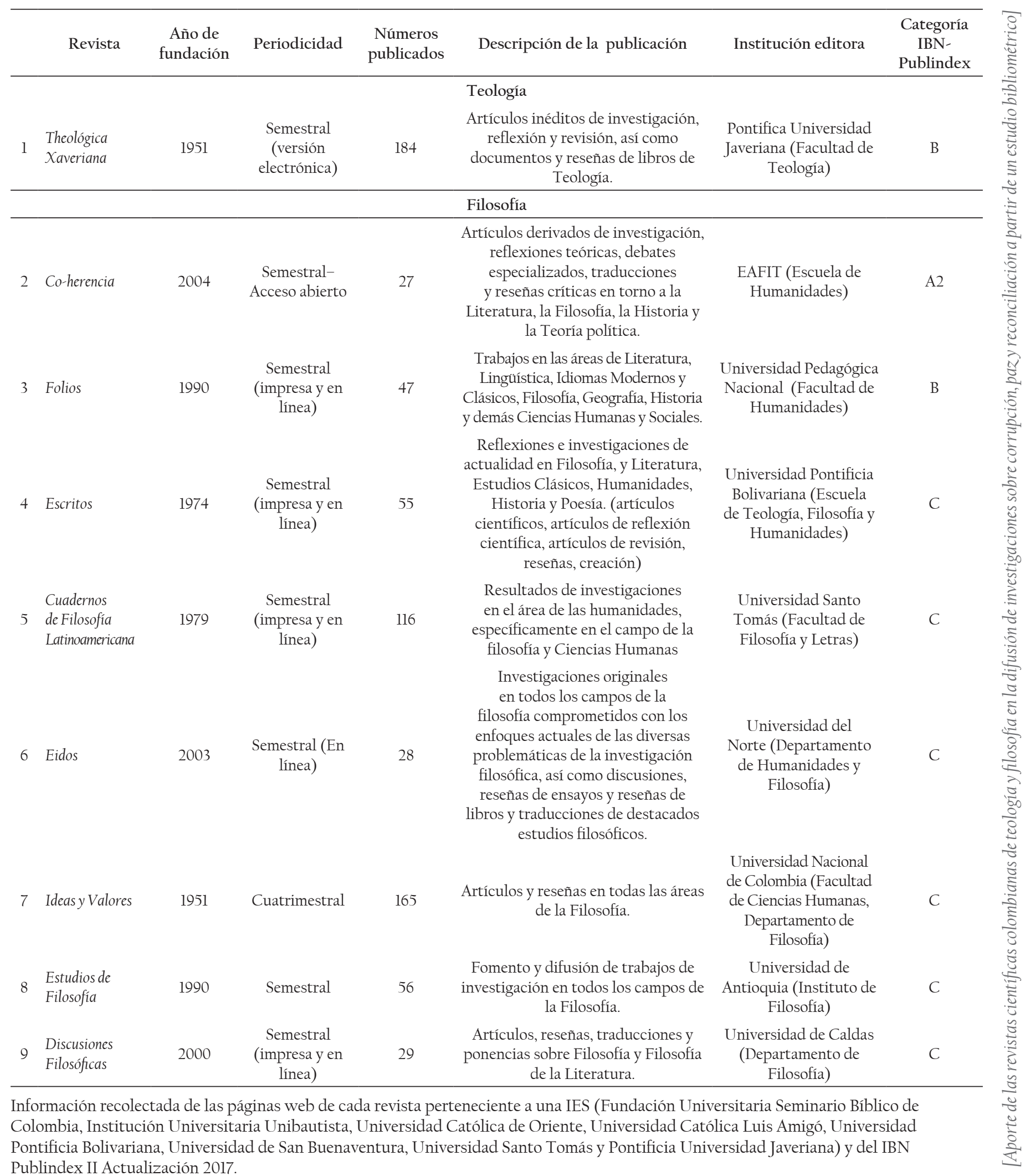

Fuente: adaptado de Ramírez et al., 2012. 
Tabla 3. Revistas científicas de teología y filosofía en Colombia, visibilidad e impacto.

\begin{tabular}{|c|c|c|c|c|c|c|}
\hline & Revista & Categoría IBN-Publindex & IBC-SJR-Scopus & FI & H-Scopus & h-5 (2011-2015)- Google Scholar Metrics \\
\hline \multicolumn{7}{|c|}{ Teología } \\
\hline 1 & $\begin{array}{l}\text { Theológica } \\
\text { Xaveriana }\end{array}$ & B & Q3 & 0,101 & 1 & 5 \\
\hline \multicolumn{7}{|c|}{ Filosofía } \\
\hline 2 & Co-herencia & A2 & Q2 & 0,110 & 2 & 7 \\
\hline 3 & Folios & B & --- & --- & --- & 6 \\
\hline 4 & Escritos & C & --- & --- & --- & 4 \\
\hline 5 & $\begin{array}{l}\text { Cuadernos } \\
\text { de Filosofia } \\
\text { Latinoamericana }\end{array}$ & C & --- & & --- & 4 \\
\hline 6 & Eidos & C & Q4 & 0,100 & 1 & 2 \\
\hline 7 & Ideas y Valores & C & Q4 & 0,100 & 2 & 5 \\
\hline 8 & Estudios de Filosofía & C & --- & --- & --- & 4 \\
\hline 9 & $\begin{array}{l}\text { Discusiones } \\
\text { Filosóficas }\end{array}$ & C & Q4 & 0,100 & 1 & 4 \\
\hline
\end{tabular}

Información recolectada de las páginas web de cada revista, de la base de datos de SJR: http://www.scimagojr.com/journalrank.php y de la base de datos de Google Scholar: https://scholar.google.es/citations?view_op=metrics_intro\&hl=es con una ventana de observación 2015-2017.

Fuente: elaboración propia.

La tabla 3 muestra que, de las nueve revistas indexadas, el 11,1\% (1) está en el cuartil Q2 de SJR, el 11,1\% (1) en el cuartil Q3, el 33,3\% (3) en el cuartil Q4 y el 44,4\% restante (4) sin cuartil. El factor de impacto va del rango de 0,100 hasta 0,110. El 88,9\% de ellas (8) declaran las bases de datos en las cuales están indexadas, siendo las más mencionadas SciELO (8), Publindex (7), Latindex (7), Ulrich's (7), Redalyc (6), Dialnet (6), Scopus (5), EBSCO (5), CLASE (5), Fuente Académica (5), DOAJ (4), WoS (3).

Según el factor de medición de SJR (del año 2016), Colombia ocupa el puesto 50 entre 239 países del mundo, con un total de 71966 documentos. En la gran área de artes y humanidades, tiene 2966 documentos con un promedio de 6,42 citaciones por documento. De estos, 123 están en la miscelánea de artes y humanidades y 49 son de filosofía. Cuenta con 84 revistas en la base SJR, en todas las áreas del conocimiento, de las cuales, 21 pertenecen a la gran área de artes y humanidades, de ellas hay cuatro en filosofía y una en estudios religiosos (teología).
La revista Theologica Xaveriana ocupa el segundo lugar entre dos revistas del área de estudios religiosos en América Latina, siendo la primera Teología y Vida de Chile. Mientras que las revistas colombianas de filosofía ocupan respectivamente el cuarto (Co-herencia), octavo (Discusiones Filosóficas), doceavo (Eidos) y decimoctavo (Ideas y Valores) lugar entre 18 revistas del área de filosofía en América Latina.

El rango del indicador h-5 de Google Scholar Metrics, para los años 2011-2015, de las nueve revistas indexadas en el IBN-Publindex va de 7 a 2, así: el 11,1 \% (1) está en 7 , el 11,1\% (1) en 6, el 22,2 \% (2) en 5, el 44,4\% (4) en 4 , y el 11,1\% restante (1) en 2.

Una vez identificados distintos índices de impacto de las revistas científicas de teología y filosofía en Colombia, se procedió a realizar el análisis de contenido de las dos temáticas propuestas, corrupción, paz y reconciliación, cuyos resultados se muestran en las tablas 4 y 5 . 
Tabla 4. Análisis de contenido sobre la temática de corrupción.

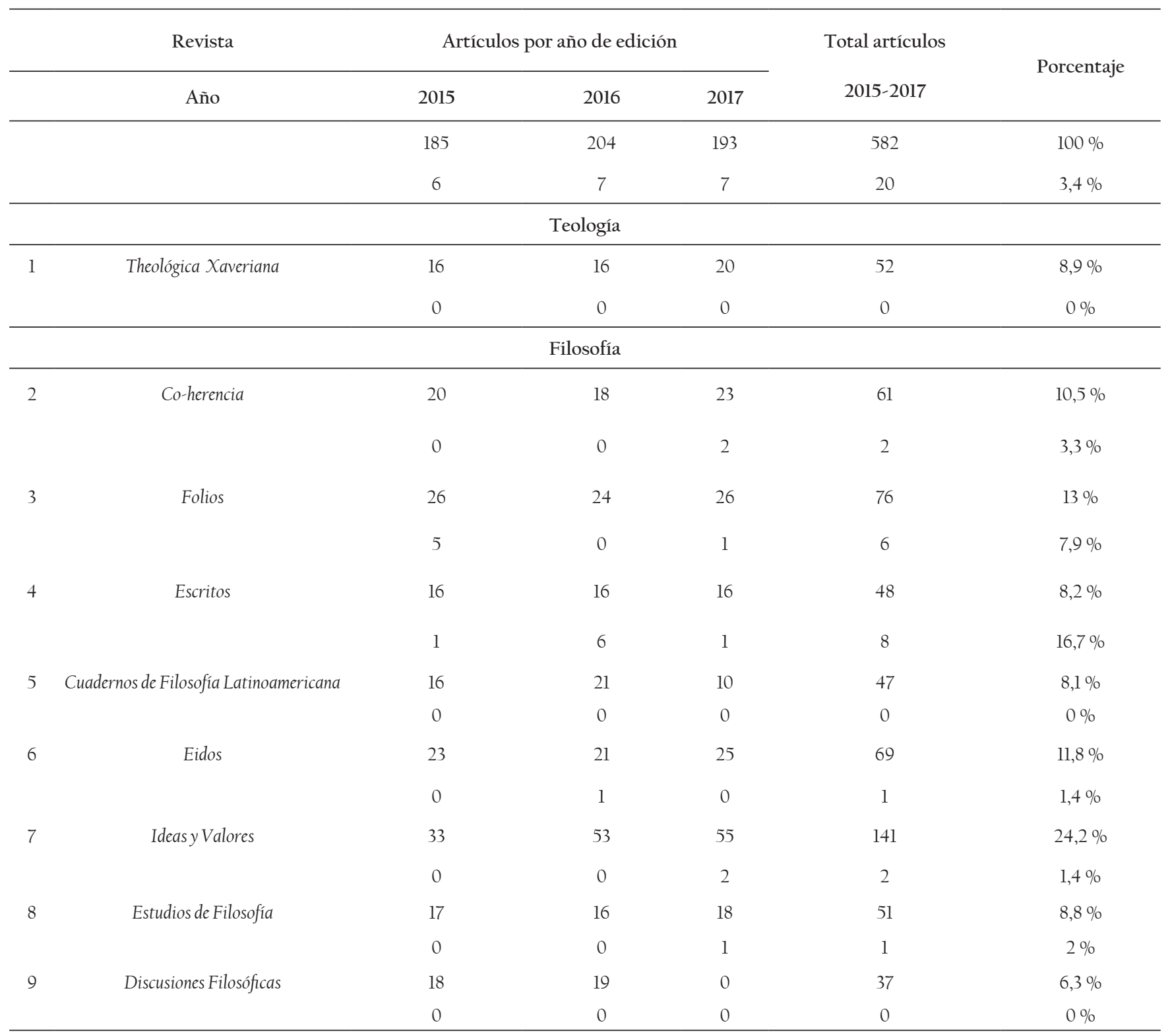

Fuente: elaboración propia. Información recolectada de las páginas web de cada revista.

En la Tabla 4, se puede observar que de las ocho revistas de filosofía, las que más aportes hicieron al tema de la corrupción en su orden son Escritos, con ocho artículos $(16,7 \%)$ de un total de 48 publicados durante la ventana de observación, seguida de la revista Folios, con seis artículos $(7,9 \%)$ de un total de 76 . Mientras Escritos tiene una gran concentración de este tema en el año 2016 (seis artículos), con énfasis en la bioética, Folios la tiene en el 2015, enfatizando la formación en ética y valores. La revista Co-herencia publicó dos artículos (3,3\%) de un total de 6l. Ideas y valores, que tiene una periodicidad cuatrimestral, aunque es la que más artículos ha publicado en la ventana de observación (141), solo aportó dos artículos (1,4\%) a esta temática. Las revistas Eidos y Estudios de Filosofía publicaron, cada una, un artículo durante los tres años revisados, mientras que Cuadernos de Filosofía Latinoamericana y Discusiones Filosóficas no publicaron ninguno. En el transcurso de los años 2015 a 2017 fue constante la publicación de artículos en la temática de corrupción. 
Tabla 5. Análisis de contenido sobre la temática de paz y reconciliación.

\begin{tabular}{|c|c|c|c|c|c|c|}
\hline & \multirow{2}{*}{$\frac{\text { Revista }}{\text { Año }}$} & \multicolumn{3}{|c|}{ Artículos por año de edición } & \multirow{2}{*}{$\begin{array}{c}\text { Total artículos } \\
\text { 2015-2017 }\end{array}$} & \multirow{2}{*}{ Porcentaje } \\
\hline & & 2015 & 2016 & 2017 & & \\
\hline & & 185 & 204 & 193 & 582 & $100 \%$ \\
\hline & & 3 & 7 & 6 & 16 & $2,7 \%$ \\
\hline \multicolumn{7}{|c|}{ Teología } \\
\hline \multirow[t]{3}{*}{1} & Theologica Xaveriana & 16 & 16 & 20 & 52 & $8,9 \%$ \\
\hline & & 0 & 0 & 0 & 0 & $0 \%$ \\
\hline & \multicolumn{6}{|c|}{ Filosofía } \\
\hline \multirow[t]{2}{*}{2} & Co-herencia & 20 & 18 & 23 & 61 & $10,5 \%$ \\
\hline & & 0 & 1 & 3 & 4 & $6,5 \%$ \\
\hline \multirow[t]{2}{*}{3} & Folios & 26 & 24 & 26 & 76 & $13 \%$ \\
\hline & & 1 & 0 & 0 & 1 & $1,3 \%$ \\
\hline \multirow[t]{2}{*}{4} & Escritos & 16 & 16 & 16 & 48 & $8,2 \%$ \\
\hline & & 0 & 0 & 0 & 0 & $0 \%$ \\
\hline \multirow[t]{2}{*}{5} & Cuadernos de Filosofía Latinoamericana & 16 & 21 & 10 & 47 & $8,1 \%$ \\
\hline & & 0 & 1 & 0 & 1 & $2 \%$ \\
\hline \multirow[t]{2}{*}{6} & Eidos & 23 & 21 & 25 & 69 & $11,8 \%$ \\
\hline & & 0 & 1 & 1 & 2 & $2,9 \%$ \\
\hline \multirow[t]{2}{*}{7} & Ideas y Valores & 33 & 53 & 55 & 141 & $24,2 \%$ \\
\hline & & 0 & 2 & 0 & 2 & $1,4 \%$ \\
\hline \multirow[t]{2}{*}{8} & Estudios de Filosofía & 17 & 16 & 18 & 51 & $8,8 \%$ \\
\hline & & 0 & 1 & 1 & 2 & $3,9 \%$ \\
\hline \multirow[t]{2}{*}{9} & Discusiones Filosóficas & 18 & 19 & 0 & 37 & $6,3 \%$ \\
\hline & & 1 & 0 & 0 & 1 & $2,7 \%$ \\
\hline
\end{tabular}

Fuente: elaboración propia. Información recolectada de las páginas web de cada revista.

En la Tabla 5, se observa que en cuanto a los aportes hechos al tema de paz y reconciliación, por parte de las revistas de filosofía, la revista Co-herencia es la que más contribuciones hizo: de un total de 61 publicaciones, aportó cuatro artículos (6,5\%), tres de ellos publicados en el 2017. Las revistas Estudios de Filosofía (con 51 publicaciones), Eidos (con 69 publicaciones) e Ideas $y$ Valores (con 141 publicaciones), aportaron cada una dos artículos a esta temática con porcentajes de 3,9\%, 2,9 \% y 1,4 \% respectivamente. Las revistas Folios, Cuadernos de Filosofía Latinoamericana y Discusiones Filosóficas publicaron cada una un artículo durante la ventana de observación. Por el contrario, Escritos no publicó ningún artículo sobre esta temática. La Tabla 5 también permite percibir que en los años 2016 y 2017 aumentó el número de publicación de artículos en la temática de paz y reconciliación.

El análisis de contenido de las temáticas corrupción, paz y reconciliación, en la ventana de observación de tres años (2015-2017), reflejados en las tablas 4 y 5, permite determinar que de un total de 582 artículos publicados, en las nueve revistas de teología y filosofía indexadas, el 3,4\% (20) corresponden al tema de corrupción y el 2,7 \% (16) al de paz y reconciliación.

La revista de teología Theologica Xaveriana publicó un total de 52 artículos en los años 2015-2017, de los cuales ninguno aborda el tema de corrupción, y solo el 5,8 \% (3) trata la temática de paz y reconciliación, un artículo por cada año observado. 


\section{Discusión y conclusiones}

Luego de que Colciencias aumentara las exigencias al implementar un nuevo sistema de clasificación del IBN-Publindex mediante criterios relacionados con gestión editorial, visibilidad e impacto, es decir, con "nivel de citación, política editorial, calidad del contenido, regularidad, accesibilidad y participación en sistemas internacionales de valoración de revistas científicas" (Colciencias, 2016, p. 20), todo esto con el fin de ajustar los estándares de calidad científica y así incrementar el impacto de las publicaciones científicas nacionales, se observó que de 542 revistas indexadas (en 2014) solo quedaron 244 (en 2017), menos del $50 \%$; y muchas de ellas bajaron su categoría de clasificación, como es el caso de la revista de teología, Theologica Xaveriana, que pasó de A2 a B (Colciencias, 2017).

Respecto al total de revistas colombianas especializadas indexadas en el IBN-Publindex, las de teología representan el 0,4\% y las de filosofía el 3,3\%, lo que muestra el bajo aporte que estas áreas del conocimiento pueden hacer a la difusión de los resultados de investigación relacionados con corrupción, paz y reconciliación.

En cuanto al impacto de las revistas, se pudo observar que la única revista de teología indexada en el IBN-Publindex está clasificada en la categoría B, según los estándares de criterios de IBN-Publindex; por otra parte, de las revistas de filosofía, solo una está clasificada en A2, mientras que la mayoría (66,7 \%) está en la categoría más baja, la C. Esta categorización influirá en el bajo reconocimiento internacional que puedan tener los investigadores y los avances de investigación publicados en estas revistas. También se hizo evidente que la mayoría de las revistas indexadas son editadas por instituciones privadas (5).

Las nueve revistas científicas de teología y filosofía indexadas demuestran buena estabilidad, la cual está reflejada en el cumplimiento de la periodicidad de las publicaciones, siendo el 88,9\% semestral y el 11,1\% cuatrimestral, y en la antigüedad de las mismas, que abarca desde sesenta y siete hasta quince años.
En lo que corresponde a la medida de posición de las revistas colombianas de teología y filosofía en relación con todas las de su área, se observó que no hay ninguna que tenga un alto FI (Q1); solo dos (22,2\%), Co-herencia y Theologica Xaveriana, están en los cuartiles medios (Q2 y Q3), tres (33,3\%) en el cuartil más bajo de SJR (Q4), y una gran proporción de ellas (44,4\%) no tienen un cuartil en SJR. La posición de estas revistas con respecto a las de su misma categoría en América Latina, en algunos casos, es buena, por ejemplo la de la revista Co-herencia, que ocupa el cuarto lugar entre 18 revistas de filosofía; pero, en otros casos, no es la mejor, ejemplo de ello es la revista Theologica Xaveriana, que, de las dos en estudios religiosos, ocupa el segundo lugar, y la de Ideas y Valores que ocupa el último lugar entre las de 18 de filosofía. Esto muestra que un buen número de las revistas colombianas de teología y filosofía tienen una baja visibilidad, reconocimiento nacional e internacional e influencia científica, lo que conduce a que los autores que divulgan los resultados de sus investigaciones en estas publicaciones científicas sean poco visibles para el resto del mundo.

$\mathrm{Al}$ analizar la difusión, las dos temáticas propuestas, corrupción, paz y reconciliación, por las revistas académicas colombianas de teología y filosofía, se identificó que los aportes que estas revistas hacen a estas temáticas, que son de gran relevancia y necesarias para la situación actual que vive el país, son muy bajos, el 3,4\% al tema de corrupción y el 2,7 \% al de paz y reconciliación. La única revista de teología, Theologica Xaveriana, en ninguno de los 52 artículos publicados entre los años 2015-2017 abordó el tema de la corrupción, y solo en un 5,8 \% (3) trató la temática de paz y reconciliación. Por su parte, de las ocho revistas de filosofía, solo dos, Escritos y Folios fueron las que más publicaron sobre corrupción, con un 16,7 \% y 7,9\% respectivamente, y la revista Co-herencia en la temática de paz y reconciliación con una representación del 6,5\%. Ideas y Valores, que tuvo el mayor número de publicaciones en la ventana de observación (141), solo aporto dos artículos de cada asunto. Se esperaría que las publicaciones en las áreas de teología y filosofía, en estos temas sobre los que el país necesita una mayor investigación y reflexión, fueran más abundantes para llevar a la comunidad académica e incluso al Estado colombiano a utilizar el resultado de sus investigaciones para reflexionar y tomar decisiones al respecto. 
El estudio bibliométrico centrado en índices de impacto de las revistas académicas colombianas de teología y filosofía y la actividad científica en los tópicos de corrupción, paz y reconciliación, ponen sobre la mesa la necesidad de evaluar el cumplimiento de los criterios de calidad de la producción científica y de la gestión editorial de las revistas colombianas en estas áreas del conocimiento, para lograr mayor visibilidad y difusión de los resultados de investigación, un intercambio de conocimientos y experiencias de tal manera que se mejoren y amplíen cada vez más las investigaciones en este campo científico, sobre todo en las dos temáticas propuestas. Para el mejoramiento de la calidad de las publicaciones científicas es importante tener en cuenta el plan estratégico que presenta Colciencias (2017) en el que propone tres objetivos específicos:

\section{Aumentar las publicaciones de los investigadores nacionales en revistas científicas incluidas en índices citacionales de alto impacto.}

\section{Fomentar acciones para fortalecer capacidades de investigadores nacionales.}

3. Aumentar la presencia de revistas científicas nacionales en los índices citacionales y bases de datos de alto impacto científico. (pp. 32-34)

Finalmente, al hacer estudios bibliométricos para evaluar el impacto de las publicaciones científicas también se debe atender a las características y dinámicas particulares de las publicaciones en el campo de las humanidades. Con respecto a esto, Toro (2017) señala cinco características distintivas de las humanidades, particularmente de teología: en primer lugar, el alcance de la investigación y el impacto, porque los asuntos investigados en teología son tan específicos que sus resultados son pertinentes solo para un grupo limitado de académicos; en segundo lugar, la tipología de los documentos publicados, ya que en teología se publica más en libros y capítulos de libros que en artículos; en tercer lugar, indica que la metodología, los métodos cualitativos y el análisis de resultados de investigación, en estas áreas del conocimiento, requiere más tiempo; en cuarto lugar, el autor menciona que las citas y referencias son más antiguas por el uso que se hace de libros y capítulos de libro, y, finalmente, se debe tener en cuenta que la investigación en esta área se hace más de forma individual que grupal y esto afecta la productividad de los autores (pp. 235-239).

\section{Referencias}

1. Barreto, M. (2017). Pensar la paz y la reconciliación en Colombia desde la experiencia de Perú: lecciones a partir del análisis de la Comisión de la verdad y reconciliación. Análisis político, 30(90), 154-174. doi: 10.15446/anpol. v30n90.68559

2. Buela-Casal, G. (2003). Evaluación de la calidad de los artículos y de las revistas científicas: Propuesta del factor de impacto ponderado y de un índice de calidad. Psicothema, 15(1), 23-35. Recuperado de https://www.unioviedo.es/reunido/index.php/PST/article/ viewFile/8133/7997

3. Cardinali, D. P. (2010). Posibles estrategias para la promoción de publicaciones científicas regionales. En II Encuentro Iberoamericano de editores científicos (p. 24). Biblioteca Nacional. Buenos Aires, 11 y 12 de noviembre de 2010. Recuperado de https:/dialnet.unirioja.es/servlet/ articulo?codigo $=3342200$

4. CNMH. (2013). iBASTA YA! Colombia: Memorias de guerra y dignidad. Informe General Grupo de Memoria Histórica. (2. ed.). Colombia: Imprenta Nacional. Recuperado de http://www.centrodememoriahistorica. gov.co/descargas/informes2013/bastaYa/basta-yacolombia-memorias-de-guerra-y-dignidad-2016.pdf

5. Colciencias. (2016). Política para mejorar el impacto de las publicaciones científicas nacionales. Documento 1601. (Versión final). Recuperado de http://www.colciencias. gov.co/sites/default/files/upload/noticias/120816vfpolitica_publindex_2.0_og_ao_miv.pdf

6. Colciencias. (2017). Informe de la Convocatoria para indexación de revistas científicas colombianas especializadas - Publindex. Recuperado de http://www.colciencias. gov.co/convocatorias/investigacion/convocatoria-paraindexacion-revistas-cientificas-colombianas

7. Cortés, I. (2016). Solidaridad comunicativa y reconocimiento: contribuciones de la filosofía para la paz al proyecto Unesco de un nuevo humanismo. Panorama, 10(19), pp. 77-83. doi: 10.15765/pnrm.vl0il9.832

8. Díaz, G. J. (2014). El índice H: una forma objetiva de evaluar la producción científica de un investigador. Revista de la Facultad de Medicina Veterinariay de Zootecnia, 61(2), 113-114. Recuperado de http://www.scielo.org.co/pdf/rfmvz/v6ln2/ v6ln2a0l.pdf

9. Fundación Universitaria Seminario Bíblico de Colombia. (ll de mayo, 2017). Página de inicio. Ventana Teológica. Recuperado de http://www.unisbc.edu.co/investigacion/ ventana-teologica 
10. Gómez, D. J. (2018). Redes de corrupción política: una revisión para el caso colombiano. Análisis político, 31(92), 180-201. doi: 10.15446/anpol.v3ln92.71106

11. González, B., Guerrero, V. P., \& Moya, F. (2010). The SJR indicator: A new indicator of journals' scientific prestige. arXiv preprint arXiv:0912.4141. Recuperado de https://arxiv.org/abs/0912.4141

12. Hicks, D., Wouters, P., Waltman, L., De Rijcke, S., \& Rafols, I. (2015). The Leiden Manifesto for research metrics. Nature, 520(7548), 429. Recuperado de https:// capes.gov.br/images/stories/download/diversos/2782015The-Leiden-Manifesto.pdf

13. Hirsch, J. E. (2005). An index to quantify an individual's scientific research output. Proceedings of the National academy of Sciences of the United States of America, 102(46), 16569-16572. Recuperado de https://www.ncbi.nlm.nih.gov/pmc/ articles/PMC1283832/pdf/pnas-0507655102.pdf

14. IBN Publindex. (20 de mayo, 2017). Búsqueda de revistas por categoría. Recuperado de http://scienti.colciencias.gov. co:8084/publindex/EnIbnPublindex/buscador.do

15. Institución Universitaria Unibautista. (04 de abril, 2017). Página de inicio. Kronos teológico. Recuperado de http:// www.unibautista.edu.co/kronos-teologico/

16. Kitchenham, B. (2004). Procedures for performing systematic reviews. Keele, UK, Keele University, 33(2004), 1-26. Recuperado de http://www.inf.ufsc.br/aldo.vw/ kitchenham.pdf

17. López, E. (2015). Teología de la historia como teología de la acción. Theologica Xaveriana, 180, 471-496. doi: 10.11144/ javeriana.tx65-180.thta

18. Miguel,S.(2011).RevistasyproduccióncientíficadeAmérica Latina y el Caribe: su visibilidad en SciELO, RedALyC y SCOPUS. Revista Interamericana de Bibliotecología, 34(2), 187199. Recuperado de https://aprendeenlinea.udea.edu.co/ revistas/index.php/RIB/article/view/10366

19. Penkova, S. (2011). Criterios nacionales e internacionales de calidad de las revistas científicas en Iberoamerica: análisis comparativo. En A. M. Cetto Kramis \& J. O. Alonso Gamboa (Comps.), Calidad e Impacto de la revista Iberoamericana (pp. 99-121). México: Facultad de Ciencias, UNAM. Recuperado de http://cort.as/-H7Z

20. Pontificia Universidad Javeriana. (10 de abril, 2017). Página de inicio. Revistas científicas Pontificia Universidad Javeriana. Recuperado de http://revistas.javeriana.edu.co/

21. Sánchez, J. C. U. (2015). Algunos apuntes sobre la corrupción. Ius et Veritas, 20(40), 370-379.
22. Ramírez, D. C., Martínez, L. C. \& Castellanos, O. F. (2012). Divulgación y Difusión del Conocimiento: Las Revistas Cientificas. Recuperado de http://www.bdigital.unal.edu. co/8394/1/9789587613346.pdf

23. Tavares, M. (2011). El peer review de las revistas científicas en Humanidades y Ciencias Sociales: políticas y prácticas editoriales declaradas. Revista Española de Documentación Científica, 34(2), 141-164. doi: 10.3989/redc.2011.2.796

24. Toro, I. (2017). Editorial. La bibliometría y las publicaciones en Teología. Cuestiones Teológicas, 44(102), 229-239. doi: 10.18566/cueteo.v44nl02.a0l

25. Transparency International. (2017). Corruption perceptions index 2017. Recuperado de https:/www.transparency.org/ news/feature/corruption_perceptions_index_2017

26. Transparencia por Colombia. (2017). Índice de Percepción de la Corrupción 2017: Corrupción, aún sin solución de fondo. Corporación Transparencia por Colombia. Capítulo Transparencia Internacional. Recuperado de http:// transparenciacolombia.org.co/2018/09/16/resultadoipc-2017//

27. Scimago Journal \& Country Rank. (10 de mayo, 2017). Journal Rankings. Recuperado de http://www.scimagojr. com/journalrank.php

28. Universidad Católica de Oriente. (05 de mayo, 2017). Página de inicio. Revista de Ciencias Sociales y Humanas. Kenosis. Recuperado de http://revistas.uco.edu.co/index.php/ kenosis/index

29. Universidad Católica Luis Amigó. (20 de mayo, 2017). Página de inicio. Revistas en línea. Fondo editorial - FUMLAN. Recuperado de http://www.funlam.edu.co/revistas/index. php/revistafunlam

30. Universidad Pontificia Bolivariana. (13 de mayo, 2017). Página de inicio. Revistas académicas y científicas. Recuperado de https://revistas.upb.edu.co/

31. Universidad de San Buenaventura. (02 de mayo, 2017). Página de inicio. Franciscanum. Recuperado de http://revistas. usb.edu.co/index.php/Franciscanum

32. Universidad Santo Tomás. (12 de abril, 2017). Página de inicio. Sistemas de Revistas Científicas. Análisis. Recuperado de http:// revistas.usta.edu.co/index.php/analisis 